\author{
Isaí Arturo Medina Fernández ${ }^{1}$ \\ Josué Arturo Medina Fernández², \\ Reyna Torres Obregón ${ }^{3}$ \\ Rebeca Sosa Cárdenas ${ }^{4}$ \\ George Williams Chale Pool ${ }^{5}$ \\ Lorena Chaparro-Díaz ${ }^{6}$
}

1. Máster en Enfermería. Facultad de Enfermería Dr. Santiago Valdés Galindo. Universidad Autónoma de Coahuila. México.

2. Máster en Enfermería. Centro Universitario Siglo XXI. Mérida. México.

3. Doctora en Ciencias de Enfermería. Facultad de Enfermería Dr. Santiago Valdés Galindo. Universidad Autónoma de Coahuila. México.

4. Máster en Enfermería. Facultad de Enfermería. Universidad Autónoma de Yucatán. México.

5. Licenciado en Enfermería. Facultad de Enfermería. Universidad Autónoma de Yucatán. México.

6. Doctora en Enfermería. Facultad de Enfermería. Universidad Nacional de Colombia, sede

Bogotá. Bogotá. Colombia.

*Autor para correspondencia.

Correo electrónico: josuemedinafernandez@outlook.es (Josué Arturo Medina Fernández).

\section{Actitudes hacia la vejez y actitudes hacia la sexualidad del adulto mayor en estudiantes y profesionales de enfermería}

\section{Attitudes towards old age and attitudes towards elderly's sexuality in students and nursing professionals}

\section{RESUMEN}

Objetivo: Analizar la relación entre las actitudes hacia la vejez y actitudes hacia la sexualidad del adulto mayor en profesionales y estudiantes de enfermería de Yucatán, México. Metodología: Se realizó un estudio con 212 estudiantes y profesionales de enfermería.

Se aplicó el instrumento de actitudes negativas hacia la vejez en población mexicana y el cuestionario de actitudes hacia la sexualidad en la vejez analizándose con SPSS v. 22 mediante estadística descriptiva e inferencial. Resultados: No existe relación entre actitudes hacia la vejez y sexualidad del adulto mayor $(p=0,504)$; sin embargo, se halló relación de la edad con los estereotipos negativos físicos y conductuales asociados a la vejez $(p=0,001)$, así como de las actitudes hacia la sexualidad del adulto mayor, miedo al envejecimiento en los estudiantes de enfermería $(p=0,022)$, y de la edad con miedo al envejecimiento $(p=0,041)$, y miedo al deterioro intelectual y/o abandono en los profesionales de enfermería $(p=0,042)$. Conclusión: Se hace necesaria la intervención para modificar los estereotipos en la muestra, lo que repercutirá en un mejor cuidado.

PALABRAS CLAVE: Anciano, actitud del personal de salud, actitud, sexualidad.

\section{ABSTRACT}

Objetive: Analyze the relationship between the attitudes towards old age and attitudes towards sexuality of the elderly in nursing professionals and students. Methodology: The study incorporate 212 students and nursing professionals from Mérida, Yucatán, Mexico. For the study, it applies the instrument of negative attitudes towards old age in the Mexican population and questionnaire attitudes towards sexuality in old age was analyzed with SPSS v. 22 using descriptive and inferential statistics. Results: indicate that there is no relationship between attitudes towards old age and the sexuality of the elderly $(p=.504)$, however there are a relationship between age and the stereotypes of physicists and the behaviors associated with old age (p. = .001), as well as attitudes toward the sexuality of the elderly and fear of aging in nursing students $(\mathrm{p}=.022)$ and of the age with fear of aging ( $p=.041)$ and the fear of intellectual deterioration and / or abandonment in nursing professionals $(\mathrm{p}=.042)$. Conclusion: It is necessary an intervention to modify the stereotypes in the sample, which will affect better attention care.

KEYWORDS: Aged, Attitude of Health Personnel, Attitude, Sexuality.

\section{- INTRODUCCIÓN}

El incremento de la población de adultos mayores representa un reto para los sistemas de salud por la relación que tiene el envejecimiento con el deterioro de la salud y la alta prevalencia de enfermedades crónicas no transmisibles, así como las necesidades en su atención ${ }^{1}$. Se calcula que entre 2015 y 2050 se duplicará la población adulta mayor, pasando el número de personas mayores de 60 años de 900 millones a 2000 millones, lo que representa un incremento del $12 \%$ a $22 \%{ }^{2}$.

En México, el incremento del envejecimiento en relación con su crecimiento poblacional es del 3,5\% por ańo. Las proyecciones del Consejo Nacional de Población (CONAPO) estiman que para el año 2050, 1 de cada 4 adultos serán adultos mayores, lo cual corresponde al 28\% de la población total, dato al que se agregan fenómenos como discapacidad y el enfrentamiento a las crisis vitales del envejecimiento ${ }^{3,4}$.

No obstante, el envejecimiento es un fenómeno multidimensional que involucra todos los aspectos de la vida humana, donde se experimentan cambios de orden físico, psicológico y social ${ }^{5}$. Sin embargo, la sociedad parece no haber considerado el impacto de dichos cambios y aún persisten actitudes que hacen que se tenga una imagen negativa de la vejez, lo que impide ver la gran cantidad de posibilidades de desarrollo que existe para los adultos mayores ${ }^{6}$.

A su vez, dichos cambios y actitudes pueden ser negativas o positivas en el profesional de enfermería, siendo una actitud, una predisposición 
organizada a pensar, sentir, percibir y comportarse hacia un referente $\mathrm{u}$ objeto cognitivo, relacionándolo con el comportamiento que mantenemos en torno a los objetos a que hacen referencia ${ }^{7}$. Atendiendo a lo antes referido, la presencia de creencias erróneas y negativas, es decir las actitudes negativas hacia la vejez, puede dar lugar a prácticas discriminatorias entre los profesionales y estudiantes de enfermería, siendo una responsabilidad preparar en conocimientos y habilidades y, sobre todo, actitudes adecuadas hacia una atención apropiada en la vejez ${ }^{8}$.

Por otra parte, los cambios físicos y fisiológicos en este grupo etario ocasionan la creencia de que son seres asexuados; no obstante, en la vejez se persiguen los mismos objetivos que en otras etapas de la vida: placer del contacto corporal, comunicación, dignidad y la seguridad emocional que ocasiona sentirse querido ${ }^{6}$. En muchas ocasiones, las actitudes negativas hacia la sexualidad en la vejez hacen que no se considere en el cuidado la dimensión sexual; sin embargo, la naturaleza íntima de los cuidados y el contacto cercano y constante con la paciente brinda una oportunidad para su atención; es por ello por lo que el profesional de enfermería tiene la mejor posición para proporcionar a los pacientes la oportunidad de hablar sobre sexualidad y salud sexual'.

Aunado a lo anterior, el cuidado de enfermería en el envejecimiento debe abordar todas las perspectivas del ser humano, basado en el entendimiento hacia los modelos de envejecimiento activo y exitoso, así como aplicarlos en el cuidado 5 . No obstante, es necesario crear una cultura de envejecimiento en el profesional y en los estudiantes de enfermería, dado que permitirá eliminar prejuicios y actitudes hacia la vejez y el proceso de envejecer, y aportará profesionales capaces de reflejar empatía y conocimiento de la misma, para brindar un cuidado holístico e integral ${ }^{10}$.

\section{- OBJETIVO}

Analizar la relación entre las actitudes hacia la vejez y actitudes hacia la sexualidad del adulto mayor en profesionales y estudiantes de enfermería de Yucatán, México.

\section{- MÉTODO}

\section{Tipo de investigación}

Se trata de un estudio correlacional, transversal, cuyo fin es conocer la relación entre dos o más variables en un periodo de tiempo único ${ }^{11}$. Dichos conceptos son las actitudes hacia la vejez y actitudes hacia la sexualidad del adulto mayor en profesionales y estudiantes de enfermería.

\section{Muestra, muestreo y selección de participantes}

La muestra estuvo constituida por estudiantes y profesionales de enfermería del estado de Yucatán, México. Se utilizó un muestreo no probabilístico a conveniencia; participaron en el estudio los estudiantes que cumplían con los requisitos de inclusión: cursar estudios de enfermería de nivel auxiliar, técnico general o licenciatura y que estuvieran en cualquier semestre de su nivel educativo. Se excluyó a los estudiantes menores de 18 ańos y se eliminaron aquellos que se retiraron del estudio o no cumplimentaron la totalidad de los instrumentos. En cuanto a los profesionales de enfermería, los requisitos de inclusión fueron tener grado de auxiliar de enfermería, técnico profesional de enfermería, licenciado en enfermería o con algún posgrado. Se excluyó a las personas mayores de 60 ańos y se eliminaron aquellos que no completaron la recolección de datos.

\section{Recogida de la información}

La recolección de datos se llevó a cabo con la técnica basada en la red mediante Google encuestas en el mes de mayo de 2018, encuesta que fue distribuida en diversos medios digitales. Primeramente, se aplicó por este medio el consentimiento informado; una vez aceptada la participación, se procedió a la aplicación de la cédula de datos sociodemográficos, seguido de los instrumentos que miden las actitudes hacia la vejez y actitudes hacia la sexualidad del adulto mayor.

\section{Instrumentos}

Las actitudes hacia la vejez son creencias negativas o positivas en esta etapa de la vida, la cual, al ser negativas, puede ocasionar prácticas de cuidado discriminatorias o prejuiciosas hacia el adulto mayor ${ }^{8}$. Para medir la variable antes señalada, se aplicó el instrumento llamado actitudes negativas hacia la vejez en población mexicana (CAV), con un alfa de Cronbach $=0,813$, cuyas subescalas son estereotipos negativos físicos y conductuales asociados a la vejez, miedo al propio envejecimiento, miedo al deterioro intelectual y al abandono. Está constituido por 21 reactivos, con una escala de tipo Likert de completamente en desacuerdo a completamente de acuerdo, cuya interpretación es que a mayor puntuación, mayores actitudes negativas hacia la vejez ${ }^{12}$.

Por otra parte, las actitudes hacia la sexualidad en el adulto mayor son las creencias positivas o negativas en este grupo etario relacionadas con la esfera sexual, como los prejuicios, derechos, limitaciones y mitos ${ }^{13}$. Para medir esta variable, se aplicó el cuestionario de actitudes hacia la sexualidad en la vejez con un alfa de Cronbach $=0,718$, constituido por 14 reactivos con una escala de Likert de falso a completamente verdadero, cuyas 12 preguntas apuntaban hacia los estereotipos y prejuicios sociales sobre la vejez y la sexualidad, y otras dos preguntas se enfocaban hacia las enfermedades resultantes del envejecimiento, el uso de medicamentos y sus efectos en la vida sexual del adulto mayor; no obstante, la medición fue a mayor puntuación, mayores actitudes negativas y prejuicios hacia la sexualidad en el adulto mayor ${ }^{13}$.

Se analizaron los datos mediante el software SPSS v. 22, utilizando estadística descriptiva para las variables sociodemográficas; así mismo, se determinó la normalidad en las variables cuantitativas a través de la prueba de Kolomorgov-Smirnov. Para determinar la diferencia de medias entre grupos de estudiantes y profesionales de enfermería para las actitudes antes mencionadas se utilizó la U de Mann Whitney; finalmente, para determinar las correlaciones, se utilizó la $r$ de Spearman.

El estudio fue aprobado por el Comité de Ética de la Facultad de Enfermería de la Universidad Autónoma de Coahuila. Así mismo está adherido a los códigos existentes, tomando como referencia el código de Núremberg, que rige la investigación con seres humanos bajo los argumentos del libre daño, así como la aplicación y aprobación del consentimiento informado por los participantes vía electrónica. De igual manera, se adhiere a la Norma oficial mexicana NOM-012-SSA3-2012, que establece los criterios para la ejecución de proyectos de investigación para la salud en seres humanos y a lo establecido en el reglamento de la Ley General de Salud de México en materia de investigación ${ }^{14}$.

\section{RESULTADOS}

La muestra estuvo constituida por 212 estudiantes y profesionales de enfermería provenientes de Yucatán, México, de los cuales el 74,5\% (158) fueron mujeres y el 25,5\% (54) hombres; la edad osciló entre los 18 y 70 años, con una $\bar{x}=27,96$ años $(\mathrm{DE}=8,99$ años $)$. En relación con el 
estado civil, el 70,8\% (150) eran solteros, el 17\% (36) casados, el 8,5\% (18) unión libre y un $3,8 \%$ (8) divorciados. Con respecto a la religión, el $76,9 \%$ (163) son católicos, el 8,5\% cristianos, el 7,5\% (16) refieren no sentirse apegados a alguna religión y el 7\%, otras religiones (15).

De los 212 participantes, el 51,4\% (109) son profesionales de enfermería y el 48,6\% (103) son estudiantes y pasantes de enfermería. En relación con los estudiantes de pregrado, el 9,70\% (10) son de nivel técnico general y el 90,3\% (93) son del nivel licenciatura, y se encuentran cursando entre el primer y el octavo semestre. Así mismo, al preguntarles si han cursado alguna asignatura de cuidado al adulto mayor, el 93,20\% (96) respondió afirmativamente.

Con respecto a los profesionales de enfermería, el 55,96\% (61) tienen grado de licenciatura, el 33,02\% (36) posgrado, el 8,73\% (9) técnicos generales y el 2,75\% (3) auxiliares. Al preguntar por el tiempo en años de labor en el área de enfermería, este osciló entre 0 y 47 años, con una $\overline{\mathrm{x}}=9,32$ años ( $\mathrm{DE}=9,09$ años). Así mismo, el 13,76\% (15) trabaja en el primer nivel, el 60,55\% (66) en el segundo y el tercer nivel, el $11 \%$ (12) en docencia y el 12,84\% (12) de manera independiente.

Por otra parte, la muestra del estudio obtuvo una puntuación de las actitudes hacia la vejez entre 23 y 74, con una $\bar{x}=40,87$ (DE = 11,24 puntos); no obstante, las subescalas de estereotipos negativos físicos y conductuales obtuvo una $\overline{\mathrm{x}}=18,79(\mathrm{DE}=5,09)$, el miedo al propio envejecimiento la $\overline{\mathrm{x}}=8,16(\mathrm{DE}=3,46)$ y el miedo al deterioro intelectual y abandono obtuvo una $\overline{\mathrm{x}}=13,91(\mathrm{DE}=3,87)$. Con respecto a las actitudes de la sexualidad en el adulto mayor, osciló entre 18 y 50 puntos, con una $\bar{x}=34,53(\mathrm{DE}=5,66)$. Se halló una diferencia significa- tiva de las actitudes hacia la sexualidad, vejez y la subescala de estereotipos entre los estudiantes de enfermería y profesionales de enfermería (tabla 1).

Se realizó la prueba de kolmogorov-Smirnov, obteniendo una distribución normal para la variable de actitudes hacia la sexualidad del adulto mayor $(p>0,05)$ y una distribución no normal para las variables actitudes hacia la sexualidad y sus subescalas, así como edad, semestre cursados y tiempo trabajando. Posteriormente, se realizan correlaciones de Spearman para la muestra (tabla 2), estudiantes (tabla 3) y profesionales de enfermería (tabla 4).

La tabla 2 indica que no existe, en la muestra, relación entre las actitudes hacia la vejez y la sexualidad en los adultos mayores $(p=0,754)$; sin embargo, la edad se relacionó con los estereotipos negativos físicos y conductuales asociados a la vejez $(p=0,001)$, es decir a mayor edad, mayores estereotipos negativos.

Los resultados antes mencionados indicaron que no existe una relación entre las actitudes hacia la vejez y la sexualidad de los adultos mayores en los estudiantes de enfermería $(p=0,526)$; no obstante, hubo relación de las actitudes hacia la sexualidad en la vejez y miedo al envejecimiento, es decir a mayor miedo al envejecimiento, menor actitudes negativas hacia la sexualidad $(p=0,022)$.

Las correlaciones en los profesionales de enfermería indicaron que no existe relación entre las actitudes hacia la vejez y sexualidad del adulto mayor $(p=0,533)$; así mismo, existe la presencia de relación de la edad con el miedo al envejecimiento $(p=0,041)$ y miedo al deterioro intelectual y/o abandono $(p=0,042)$, es decir a mayor edad, mayor miedo al envejecimiento y miedo al deterioro intelectual o abandono.

Tabla 1. Diferencia de actitudes hacia la vejez y actitudes hacia la sexualidad en estudiantes y profesionales de enfermería

\begin{tabular}{|c|c|c|c|c|c|}
\hline \multirow[t]{2}{*}{ Variables } & \multicolumn{2}{|c|}{$\begin{array}{c}\text { Estudiantes } \\
N=103\end{array}$} & \multicolumn{2}{|c|}{$\begin{array}{l}\text { Profesionales de enfermería } \\
\qquad N=109\end{array}$} & \multirow{2}{*}{$p$} \\
\hline & $\overline{\mathbf{x}}$ & DE & $\overline{\mathbf{x}}$ & DE & \\
\hline Actitudes hacia la sexualidad del adulto mayor & 33,49 & 5,14 & 35,52 & 5,93 & 0,009 \\
\hline $\begin{array}{l}\text { Actitudes hacia la vejez } \\
\text { Estereotipos } \\
\text { Miedo al envejecimiento } \\
\text { Miedo al deterioro intelectual y al abandono }\end{array}$ & $\begin{array}{c}39,08 \\
17,28 \\
8,30 \\
13,50\end{array}$ & $\begin{array}{l}10,47 \\
5,05 \\
3,38 \\
3,96\end{array}$ & $\begin{array}{c}41,58 \\
20,22 \\
8,04 \\
14,31\end{array}$ & $\begin{array}{l}11,72 \\
6,30 \\
3,55 \\
3,76\end{array}$ & $\begin{array}{l}0,033 \\
0,000 \\
0,493 \\
0,117\end{array}$ \\
\hline
\end{tabular}

$p=$ nivel de significación. ${ }^{a} p<0,05,{ }^{b} p<0,001$.

Fuente: $\mathrm{CAV}$ y $C A E V, n=212$.

Tabla 2. Correlación de actitudes hacia la vejez y sexualidad del adulto mayor de la muestra

\begin{tabular}{|c|c|c|c|c|c|c|}
\hline Variables & 1 & 2 & 3 & 4 & 5 & 6 \\
\hline 1. Actitudes hacia la sexualidad & 1 & & & & & \\
\hline 2. Actitud hacia la vejez & $\begin{array}{c}-0,022 \\
p=0,754\end{array}$ & 1 & & & & \\
\hline 3. Estereotipos negativos físicos y conductuales & $\begin{array}{c}0,042 \\
p=0,540\end{array}$ & $\begin{array}{c}0,898^{a} \\
p=0,000\end{array}$ & 1 & & & \\
\hline 4. Miedo al envejecimiento & $\begin{array}{c}-0,99 \\
p=0,212\end{array}$ & $\begin{array}{c}0,726^{b} \\
p=0,000\end{array}$ & $\begin{array}{c}0,524^{b} \\
p=0,000\end{array}$ & 1 & & \\
\hline 5. Miedo al deterioro intelectual y al abandono & $\begin{array}{c}-0,023 \\
p=0,736\end{array}$ & $\begin{array}{c}0,882^{b} \\
p=0,000\end{array}$ & $\begin{array}{c}0,669^{b} \\
p=0,000\end{array}$ & $\begin{array}{c}0,558^{b} \\
p=0,000\end{array}$ & 1 & \\
\hline 6. Edad & $\begin{array}{c}0,117 \\
p=0,090\end{array}$ & $\begin{array}{c}0,100 \\
p=0,147\end{array}$ & $\begin{array}{c}0,228 \\
p=0,001^{b}\end{array}$ & $\begin{array}{c}-0,072 \\
p=0,296\end{array}$ & $\begin{array}{c}0,032 \\
p=0,644\end{array}$ & 1 \\
\hline
\end{tabular}


Tabla 3. Correlación de actitudes hacia la vejez y actitudes hacia la sexualidad del adulto mayor en los estudiantes de enfermería

\begin{tabular}{|c|c|c|c|c|c|c|}
\hline 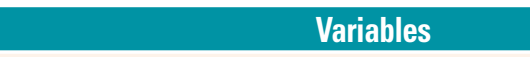 & 1 & 2 & 3 & 4 & 5 & 6 \\
\hline 1. Actitudes hacia la sexualidad & 1 & & & & & \\
\hline 2. Actitud hacia la vejez & $\begin{array}{c}-0,066 \\
p=0,509\end{array}$ & 1 & & & & \\
\hline 3. Estereotipos negativos físicos y conductuales & $\begin{array}{c}0,087 \\
p=0,387\end{array}$ & $\begin{array}{c}0,894^{b} \\
p=0,000\end{array}$ & 1 & & & \\
\hline 4. Miedo al envejecimiento & $\begin{array}{c}-0,235 \\
p=0,017^{a}\end{array}$ & $\begin{array}{c}0,721^{b} \\
p=0,000\end{array}$ & $\begin{array}{c}0,524^{b} \\
p=0,000\end{array}$ & 1 & & \\
\hline 5. Miedo al deterioro intelectual y al abandono & $\begin{array}{c}-0,048 \\
p=0,630\end{array}$ & $\begin{array}{c}0,917^{b} \\
p=0,000\end{array}$ & $\begin{array}{c}0,669^{b} \\
p=0,000\end{array}$ & $\begin{array}{c}0,538^{b} \\
p=0,000\end{array}$ & 1 & \\
\hline 6. Edad & $\begin{array}{c}-0,020 \\
p=0,839\end{array}$ & $\begin{array}{c}0,080 \\
p=0,421\end{array}$ & $\begin{array}{c}0,062 \\
p=0,534\end{array}$ & $\begin{array}{c}0,148 \\
p=0,136\end{array}$ & $\begin{array}{c}0,025 \\
p=0,802\end{array}$ & 1 \\
\hline
\end{tabular}

$p=$ nivel de significación. ${ }^{\mathrm{a}} p<0,05,{ }^{\mathrm{b}} p<0,001$.

Fuente: $\mathrm{CAV}$ y $C A E V, n=103$

Tabla 4. Correlación de actitudes hacia la vejez y actitudes hacia la sexualidad del adulto mayor en los profesionales de enfermería

\begin{tabular}{|c|c|c|c|c|c|c|}
\hline Variables & 1 & 2 & 3 & 4 & 5 & 6 \\
\hline 1. Actitudes hacia la sexualidad & 1 & & & & & \\
\hline 2. Actitud hacia la vejez & $\begin{array}{c}-0,023 \\
p=0,810\end{array}$ & 1 & & & & \\
\hline 3. Estereotipos negativos físicos y conductuales & $\begin{array}{c}-0,041 \\
p=0,671\end{array}$ & $\begin{array}{c}0,906^{b} \\
p=0,000\end{array}$ & 1 & & & \\
\hline 4. Miedo al envejecimiento & $\begin{array}{c}-0,030 \\
p=0,758\end{array}$ & $\begin{array}{c}0,763^{b} \\
p=0,000\end{array}$ & $\begin{array}{c}0,438^{b} \\
p=0,000\end{array}$ & 1 & & \\
\hline 5. Miedo al deterioro intelectual y al abandono & $\begin{array}{c}-0,038 \\
p=0,698\end{array}$ & $\begin{array}{c}0,831^{b} \\
p=0,000\end{array}$ & $\begin{array}{c}0,781^{b} \\
p=0,000\end{array}$ & $\begin{array}{c}0,538^{b} \\
p=0,000\end{array}$ & 1 & \\
\hline 6. Edad & $\begin{array}{c}-0,074 \\
p=0,446\end{array}$ & $\begin{array}{c}-0,101 \\
p=0,294\end{array}$ & $\begin{array}{c}0,049 \\
p=0,610\end{array}$ & $\begin{array}{c}-0,196 \\
p=0,041^{a}\end{array}$ & $\begin{array}{c}-0,196 \\
p=0,042^{a}\end{array}$ & 1 \\
\hline
\end{tabular}

$p=$ nivel de significación. ${ }^{a} p<0,05,{ }^{b} p<0,001$

Fuente: CAV y CAEV, $n=109$

Por otra parte, no hay diferencia entre medias de las actitudes de la sexualidad y actitudes hacia la vejez con las variables sexo, estado civil, religión, nivel de estudios de los estudiantes y grado de los profesionales de enfermería $(p<0,05)$.

\section{DISCUSIÓN}

El objetivo de esta investigación fue analizar la relación de las actitudes hacia la vejez y actitudes hacia la sexualidad del adulto mayor en profesionales y estudiantes de enfermería de Yucatán, México.

De los resultados se evidencia mayor cantidad de mujeres $(74,5 \%)$ de la población total participante, tanto de estudiantes como de profesionales, estado civil con mayoría de solteros, y edades de los participantes entre 18 y 70 ańos, teniendo una minoría en la cantidad de hombres debido a que en nuestro entorno sociocultural se asignan diferentes características (valores, atributos, posibilidades de desarrollo personal y social) a los individuos en función de su pertenencia al género femenino o masculino, lo que se atribuye a algunos empleos, dándose una fuerte identificación de la medicina con el estereotipo de género masculino y de la enfermería con el del género femenino ${ }^{15}$.

Con respecto a la actitud de los estudiantes frente a la vejez, se encontró una $\overline{\mathrm{x}}=39,08, \mathrm{DE}=10,47$, considerándose mayormente con actitud negativa. Esto coincide de una forma más puntual con un estudio realizado en Perú, en el cual el $27 \%$ obtuvo una aceptación con intensidad moderada hacia el envejecimiento ${ }^{16}$; en México el $47 \%$ tiene una actitud negativa ${ }^{17}$ y en Estado Unidos y Espańa se consideraron actitudes positivas, siendo más alto en estudiantes con mayor grado en la carrera $(p<0,001)^{18,19}$.

En relación con la actitud del profesional de enfermería hacia la vejez se obtuvo una $\bar{x}=41,58, D E=5,05$, resultando mayormente con actitudes negativas, considerándose de igual forma los resultados elaborados por Sampén y colaboradores en donde se encontró que el 59\% del personal de salud del primer nivel de atención presentó actitudes positivas hacia el envejecimiento ${ }^{20}$, siendo más elevado el porcentaje $(97,6 \%)$ en los trabajadores de salud de la Emergencia en Lima, Perú ${ }^{21}$.

Los hallazgos apoyan investigaciones que indican que los estudiantes de enfermería a menudo carecen de los conocimientos acerca de las personas mayores y necesitan oportunidades para desarrollar actitudes positivas hacia ellos en comparación con los profesionales de enfermería, que conforme más contacto tienen, menores actitudes negativas tendrán hacia el adulto mayor y el envejecimiento ${ }^{18}$; sin embargo, en este estudio, los profesionales de enfermería obtuvieron una mayor media; dichos resultados pueden ser debidos a la presencia de desgaste profesional en el cuidado al adulto mayor.

En otro punto se encontró que los resultados relativos a la actitud hacia la sexualidad por parte de los estudiantes fueron en su mayoría 
actitudes negativas $(\overline{\mathrm{x}}=33,49, \mathrm{DE}=5,14)$; esto coincide con las actitudes negativas referidas en Chile ${ }^{22}$; desde otro enfoque más cualitativo en Perú, se concluyó que poseen deficiencias en la flexibilidad de cambio de opinión y percepción negativa sobre el proceso de envejecimiento ${ }^{23}$.

De igual forma, en otro estudio se evidenció que los estudiantes de enfermería entrevistados tienen vergüenza al tocar el tema de la sexualidad del adulto mayor guiados por los prejuicios implantados por la sociedad, siendo para este autor necesaria la información en atención de la salud sexual del adulto mayor ${ }^{23}$. A partir de esto se considera que la imagen de la sexualidad de las personas mayores va ligada con las atribuciones negativas, a las condiciones físicas y habilidades sociales; esta imagen coincide con otro estudio donde los estudiantes tienden a creer que la vitalidad física decae con los ańos ${ }^{22}$.

Dentro de este punto, al caracterizar las actitudes de la sexualidad en el adulto mayor desde el punto de vista de los profesionales, se observó una $\overline{\mathrm{x}}=35,52, \mathrm{DE}=5,93$, encontrándose en mayor proporción actitudes negativas. Esto es debido a que los profesionales con menos experiencia manifiestan sentirse poco preparados y con cierta vergüenza o falta de confianza en sí mismos para actuar sobre temas de sexualidad debido a la falta de formación para la educación sexual, poca experiencia, creencias personales y religiosas sobre la sexualidad o miedo a que el paciente se sienta ofendido ${ }^{24}$.

No se encontró correlación entre las actitudes hacia la sexualidad con las actitudes hacia la vejez; esto puede ser debido a que las representaciones que posee la sociedad sobre el adulto mayor determinan la forma del cuidado; de este modo, una visión negativa del envejecimiento puede desencadenar una actitud paternalista hacia este grupo frente a una actitud más realista sobre el proceso de envejecimiento ${ }^{25}$.

De este modo se ha demostrado que la actitud de los profesionales y estudiantes de enfermería puede tener un impacto en la actitud y comportamiento de los adultos mayores con respecto a la sexualidad y el envejecimiento, a pesar de que se tenga o no la capacitación sobre la sexualidad en el adulto mayor ${ }^{23}$; así mismo, es necesario modificar las actitudes ne- gativas hacia el adulto mayor y enseñar que la atención al adulto mayor en todos sus contextos es un tema prioritario.

Se sugiere considerar, en futuras investigaciones, la variable de síndrome de desgaste profesional, para observar si puede influir sobre las actitudes, así como la presencia de alguna condición crónica y actitudes propias a la sexualidad.

\section{- CONCLUSIÓN}

Los resultados indicaron que no existe relación entre actitudes hacia la vejez y sexualidad del adulto mayor, tanto en la muestra como por grupo; sin embargo, se identificó que hay una diferencia significativa de dichas variables entre los profesionales y estudiantes de enfermería.

No obstante, se halló relación entre la edad y los estereotipos negativos físicos y conductuales asociados a la vejez en la muestra, así como la relación de las actitudes hacia la sexualidad del adulto mayor y miedo al envejecimiento en los estudiantes de enfermería; y por último, se encontró relación de la edad con el miedo al envejecimiento y miedo al deterioro intelectual y/o abandono en los profesionales de enfermería.

Lo anteriormente referido resalta la necesidad de realizar intervenciones para reforzar las actitudes positivas en los estudiantes y profesionales de enfermería; en el caso de los estudiantes, se deben agregar o mejorar las temáticas de estereotipos y prejuicios en los adultos mayores. La importancia de desarrollar las actitudes es porque forman parte del aprendizaje, el cual repercutirá en una mejor atención profesional en el futuro.

En el caso de los profesionales de enfermería es necesario capacitar mediante educación para una atención integral al adulto mayor, sin estereotipos y prejuicios que puedan impactar en el estado de salud del paciente.

\section{Conflicto de intereses}

Los autores declaran no tener ningún conflicto de intereses.

\section{- BIBLIOGRAFÍA}

1. Alvarado-García A, Lamprea-Reyes L, Murcia-Tabares K. La nutrición en el adulto mayor: una oportunidad para el cuidado de enfermería. Enfermería Univ [Internet]. 2017 [citado 13 de abri de 2018];14(3):199-206. Disponible en: http://linkinghub.elsevier. com/retrieve/pii/S1665706317300398

2. Organización Mundial de la Salud. La salud mental y los adultos mayores [Internet]. 2018 [citado 13 de abril de 2018]. Disponible en: http://origin.who.int/mediacentre/factsheets/fs381/es/

3. Aguilla E, Manquing M, Kapteyn A, Pierson A. Envejecer En México: Condiciones de Vida y Salud. [Internet] 2011 [citado 13 de abril de 2018]. 1-128 p. Disponible en: http://coespo qroo.gob. $\mathrm{mx} /$ Descargas/doc/15\%20ENVEJECIMIENTO\%2OPOBLACIONAL/ ENVEJECER\%20EN\%20M\%C3\%89XICO.pdf

4. Secretaría de Salud. Perfil Epidemiológico del Adulto Mayor en México 2010. [Internet] 2011 [citado 14 de abril de 2018]. 1936 p. Disponible en: https://epidemiologiatlax.files.wordpress $\mathrm{com} / 2012 / 10 / p$ epi del adulto mayor_en mexico 2010.pdf

5. Alvarado A, Salazar Á. Análisis del concepto de envejecimiento. Gerokomos [Internet] 2014 [citado 22 de abril de 2018];25(6):57-62 Disponible en: http://scielo.isciii.es/pdf/geroko/v25n2/revision1.pdf

6. Granadillo L, Árraga M, Sierraalta B. Actitud hacia la vejez de estudiantes de gerontología. Rev la Univeridad del Zulia. 2015;15(6):43-57

7. Kerlinger F, Lee H. Investigación del comportamiento: Métodos de investigación en ciencias sociales. México: MC Graw Hill; 2002.

8. Sarabia C, Castañedo C. Modificación de estereotipos negativos en la vejez en estudiantes de enfermería. Gerokomos [Internet]. 2015 [citado 22 de abril de 2019];26(1):10-2. Disponible en: http://scielo.isciii.es/scielo.php?script=sci_arttext\&pi$d=\$ 1134-928 \times 2015000100003$
9. Southard N, Keller J. The Importance of Assessing Sexuality. Clin J Oncol Nurs. 2008;13(2):213-8.

10. Guerrero-Castañeda R, Ojeda M. El envejecimiento desde la percepción de enfermería. Rev Enfermería Actual en Costa Rica [Internet]. 2017 [citado 23 de abril de 2018];32. Disponible en: http://www.scielo. sa.cr/pdf/enfermeria/n32/1409-4568-enfermeria-32-00155.pdf

11. Hernández R, Fernández C, Baptista P. Metodología de Investigación. México: Mc GrawHill; 2010. p. 4, 80 y 81

12. Hernández-Pozo M, Torres M, Coronado O, Herrera A, Castillo P. Sánchez A. Actitudes negativas hacia la vejez en población mexicana: Aspectos psicométricos de una escala. En: evaluación en psicogerontología. México. Moderno EM; 2009;. p. 1-16.

13. Orozco I, Rodríguez D. Prejuicios y actitudes hacia la sexualidad en la vejez. Psicol y Cienc Soc. 2006:8(001):3-10.

14. NOM-012-SSA3-2012 Que establece los criterios para la ejecución de proyectos de investigación para la salud en seres humanos Pub. Diario Oficial de la Federación (2012).

15. Mart B. Minoría de hombres en la profesión de enfermería. Re flexiones sobre su historia, imagen y evolución en España. Enfermería Glob. 2015;14(37):328-34.

16. Tipacti K. Actitud de los estudiantes de enfermería de la universidad San Juan Bautista frente al cuidado biopsicosocial en adultos mayores hospitalizados chincha [tesis]. Universidad Privada San Juan Bautista; 2018.

17. Hernández I, Moguel 0, Herández M, Lumbreras I, Banderas J. Actitud de los estudiantes de enfermería hacía los adultos mayores evaluada con las escalas de Osgood y Kogan. Enfermería Univ. 2018;15(2):147-58

18. Egilegor XH, Gorostidi XZ, Lasa MB, Cascante XS. Mitos y realidades sobre la vejez y la salud en los estudiantes de enfermería. Ge- rokomos [internet]. 2003 [citado 10 de agosto de 2018]:14(4):18894. Disponible en: https://www.gerokomos.com/wp-content/ uploads/2015/01/14-4-2003-188-rincon.pdf

19. Ryan M, McCauley D. We built it and they did not come: knowledge and attitudes of baccalaureate nursing students toward the elderly. J N Y State Nurses Assoc [Internet]. 2004.[citado 10 de agosto de 2018];35(2):5-9. Disponible en: http://europepmc.org/ abstract/MED/15884479/reload=0

20. Celis JDS, Pinedo LFV, Vélez CD, Rodríguez TT, Saavedra PJO. Validación de la Escala de Actitudes hacia el Adulto Mayor de Kogan y evaluación de las Actitudes hacia el adulto mayor por parte del personal de salud del primer nivel asistencial. Acta Médica Perú. 2012;29(3):148-54

21. Ortiz Saavedra PJ, Tello Rodríguez T, Pérez Correa ME, Varela Pinedo LF, Chávez Jimeno HA. Actitudes hacia la atención del adulto mayor por parte del personal que labora. Acta Med Per. 2013;30(2):75-8.

22. Urquiza A, Thumala D, Carthalifaud M, Ojeda A, Vogel N. Sexualidade na terceira idade. A imagem de jovens universitários Sexuality in the third age. The image of the university young people. Ponto-e-Vírgula. 2008;(4):358-74.

23. Sangurima G, Velasco S. Percepción de la sexualidad del adulto mayor en estudiantes de enfermería de la universidad de cuenca [tesis]. Universidad de Cuenca; 2016.

24. Royal College of Nursing. Older people in care homes: sex, sexuality and intimate relationships. London; [internet] 2011 [citado 2 de septiembre de 2018]. Disponible en: https://www.rcn.org.uk/ professional-development/publications/pub-007126

25. Pedrero-García E, Moreno-Crespo P Moreno-Fernández 0 . Sexualidad en Adultos Mayores: Estereotipos en el Alumnado Universitario del Grado de Educación Primaria. Form Univ. 2018;11(2):77-86. 